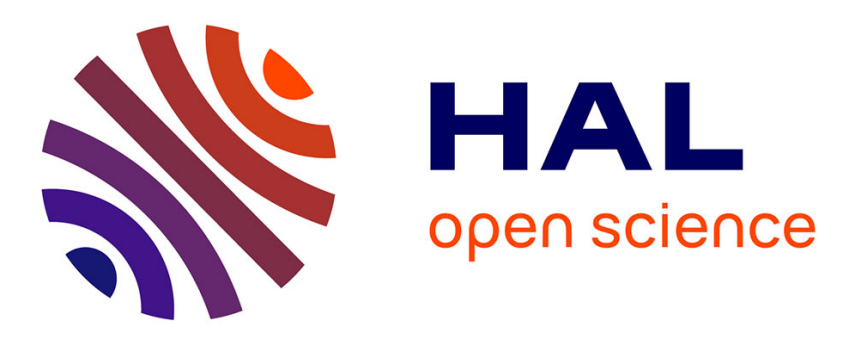

\title{
Kalman Filter for Discrete-Time Stochastic Linear Systems Subject to Intermittent Unknown Inputs
}

\author{
Jean-Yves Keller, Dominique Sauter
}

\section{To cite this version:}

Jean-Yves Keller, Dominique Sauter. Kalman Filter for Discrete-Time Stochastic Linear Systems Subject to Intermittent Unknown Inputs. IEEE Transactions on Automatic Control, 2013, 58 (7), pp.1882-1887. 10.1109/TAC.2013.2264739 . hal-00846428

\section{HAL Id: hal-00846428 \\ https://hal.science/hal-00846428}

Submitted on 19 Jul 2013

HAL is a multi-disciplinary open access archive for the deposit and dissemination of scientific research documents, whether they are published or not. The documents may come from teaching and research institutions in France or abroad, or from public or private research centers.
L'archive ouverte pluridisciplinaire HAL, est destinée au dépôt et à la diffusion de documents scientifiques de niveau recherche, publiés ou non, émanant des établissements d'enseignement et de recherche français ou étrangers, des laboratoires publics ou privés. 


\title{
Kalman filter for discrete-time stochastic linear systems subject to intermittent unknown inputs
}

\author{
J.Y. Keller, and D. Sauter, Member, IEEE
}

\begin{abstract}
State estimation of stochastic discrete-time linear systems subject to persistent unknown inputs has been widely studied but only few works have been dedicated to the case where unknown inputs may be simultaneously or sequentially active or inactive. In this paper, a Kalman filter approach is proposed for state estimation of systems with unknown intermittent inputs. The design is based on the minimisation of the trace of the state estimation error covariance matrix under the constraint that the state estimation error is decoupled from the unknown inputs corrupting the system at the current time. The necessary and sufficient stability conditions are established considering the upper bound of the prediction error covariance matrix.
\end{abstract}

Index Terms - Kalman filter, intermittent unknown inputs, linear system, covariance matrices.

\section{INTRODUCTION}

K ALMAN filtering plays an essential role in systems theory and has found a wide range of applications [19] and [29]. The problem of optimal state filtering in the presence of persistent unknown inputs, representing unknown disturbances or unmodeled dynamics, has received considerable attention in the last three decades. The most common approach consists in representing the unknown inputs as a deterministic or stochastic bias model, augment the state of the system with the bias, and apply the Kalman filter to the augmented state model of the system and finally reduce the computational time requirement [10], [1], [20], [17], [18] and [23]. When no prior information about the unknown input is available, an optimal recursive state filter is presented in [24] so that the state estimation error is decoupled from unknown inputs. Another approach which consists in transforming a standard system with unknown inputs into a singular system without unknown inputs was introduced in [6]. Other optimal filters closer to the standard Kalman filter have been derived by minimising the estimation error covariance matrix with respect to a reduced state feedback gain representing the

Manuscript received May 12, 2011; revised September 25, 2011 and March 21, 2012; Accepted July 10, 2012 ???

D. Sauter is with University of Lorraine, CRAN-CNRS UMR 7039, BP 239, 54506 Vandoeuvre les Nancy, France (phone: +33(0)3 836844 67; fax: +33(0)3 836844 62; e-mail: dominique.sauter@ univ-lorraine.fr).

J. Y. Keller is with Universityof Lorraine, CRAN-CNRS UMR 7039, BP 239, 54506 Vandoeuvre les Nancy, France. degrees of freedom in the design [5], [7] and [15]. The closely related problem of joint input and state estimation for linear discrete-time systems, which are of great importance for Fault Tolerant Control (FTC) when each component of the unknown inputs vector represents actuator or component faults [2], has been recently studied in [11], [12] and [8]. Note that the unknown inputs decoupling constraint used in [24] can be viewed as a limit case of a more general assignment taken into account in the design of stochastic detection filters [26], [22] and [21] for Fault Detection and Isolation (FDI).

Recent technological advances are revolutionizing our ability to build massively distributed Networked Control Systems (NCS). They present challenging problems arising from the fact that sensors, actuators and controllers exchange information via a digital communication network. The most common network effect can be categorized into additional network induced delays, packets losses, jitters,... In the framework of this paper, we are mainly concerned by packets losses, leading to intermittent data transmission. The state of the art on design control systems that take into account the effects of packet loss and packet delay in networked control systems have been surveyed in [16]. In particular, Kalman filtering with random lost of observations represented by Markovian or Bernoulli processes has been studied in [9] and [30], extended later to include both packet loss and random delay [27], [28] and more recently in [31] when the arrival of observations is driven by a semi-Markov chain.

This paper proposes a state filtering strategy for discretetime linear stochastic systems in the case where the arrival of unknown inputs is described by a known binary sequence. Instead of using the parameterized approach proposed in [7] which requires to pre-compute off-line the structure of the state feedback gain for each combinatorial situation of the binary sequence, the intermittent unknown inputs decoupling constraint will be parameterized from two constant size matrices, called the free part and the constrained part of the filter gain. The constrained gain, structurally dependent on the binary sequence, will be linked to the intermittent unknown inputs estimator. From a two-stage optimisation strategy very similar to those described in [10], the free gain and the constrained gain will be both used to minimize the trace of the state estimation error covariance matrix and the trace of the unknown inputs estimation error covariance matrix. The obtained filter, called the Intermittent unknown Input Kalman Filter (IIKF), will take the form of a standard Kalman filter 
updated online from the intermittent unknown inputs estimation. The stability of the IIKF will be studied on the upper bound of the prediction error covariance matrix via the stability and convergence conditions established for the Unknown Input Kalman Filter (UIKF) designed under persistent unknown inputs.

The paper is organised as follows: Section 2 explains the state filtering problem in the presence of intermittent unknown inputs. Section 3 solves the state filtering problem and studies the filter's stability before conclusions in Section 4.

\section{Problem Statement}

Let us consider the following linear discrete-time stochastic systems

$$
\begin{aligned}
& x_{k+1}=A x_{k}+B u_{k}+F d_{k}^{\theta}+w_{k} \\
& y_{k}=C x_{k}+v_{k}
\end{aligned}
$$

where $x_{k} \in \Re^{n}, \quad u_{k} \in \Re^{d}, \quad y_{k} \in \mathfrak{R}^{m}$ and $d_{k}^{\theta} \in \mathfrak{R}^{q}$ are the state, control, measurement and unknown input vectors with $q \leq m$. Matrices $A, B, C$ and $F$ are of appropriate dimensions with $\operatorname{rank}(C F)=\operatorname{rank}(F)=q$. The process and sensor noises $w_{k} \in \mathfrak{R}^{n}$ and $v_{k} \in \mathfrak{R}^{m}$ are zero mean uncorrelated Gaussian random sequences with

$$
E\left\{\left[\begin{array}{l}
w_{k} \\
v_{k}
\end{array}\right]\left[\begin{array}{l}
w_{j} \\
v_{j}
\end{array}\right]^{T}\right\}=\left[\begin{array}{cc}
W & 0 \\
0 & I
\end{array}\right] \delta_{k, j} \text { with } W \geq 0
$$

The initial state $x_{0}$, assumed to be uncorrelated with $w_{k}$ and $v_{k}$, is a Gaussian random variable with $E\left\{x_{0}\right\}=\bar{x}_{0}$ and $P_{0}=E\left\{\left(x_{0}-\bar{x}_{0}\right)\left(x_{0}-\bar{x}_{0}\right)^{T}\right\} \geq 0$.

The term $F d_{k}^{\theta}$ in (1.a) is used to describe additive unknown inputs as for example interconnecting external inputs in the context of large scale NCS. It is further assumed that $d_{k}^{\theta}$, due to possible unreliable data transmissions, is an intermittent unknown inputs vector

$$
d_{k}^{\theta}=\left[\begin{array}{lllll}
\rho_{k}^{1} d_{k}^{1} & \ldots & \rho_{k}^{i} d_{k}^{i} & . . & \rho_{k}^{q} d_{k}^{q}
\end{array}\right]^{T}
$$

depending on the known binary variables

$$
\theta_{k}=\left\{\rho_{k}^{1}, \ldots, \rho_{k}^{i}, \ldots, \rho_{k}^{q}\right\}
$$

where $\rho_{k}^{i}=1$ when the $\mathrm{i}$ th component of $d_{k}^{\theta}$ is active and $\rho_{k}^{i}=0$ otherwise.

As illustrated by Fig. 1, $u_{k}$ represents the control signals sent by the local controller to the plant and $d_{k}^{\theta}$ the control signals sent by remote controller with $\rho_{k}^{i}=1$ when $d_{k}^{i}$ is received by the plant or $\rho_{k}^{i}=0$ when $d_{k}^{i}$ is lost through the unreliable network. The unreliable network is a multichannel network where each component of $d_{k}^{\theta}$ may be lost independently from the others. The acknowledgement signals $\theta_{k}$ indicating the status of reception/delivery (TCP for example) and the distribution matrix $F$ of $d_{k}^{\theta}$ are both assumed known to the local controller.

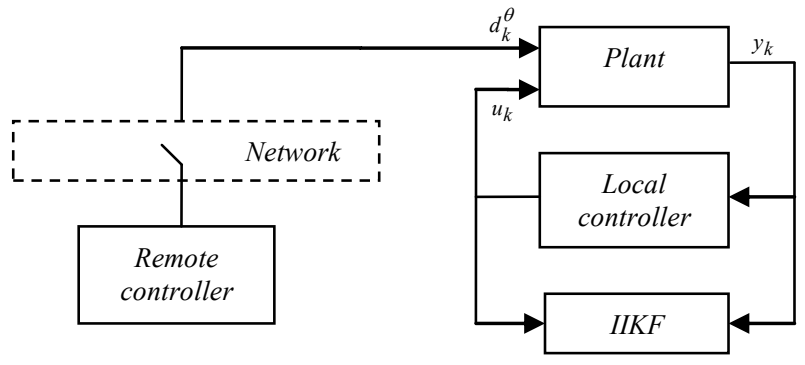

Fig. 1. Intermittent unknown inputs in NCS.

The arrival sequence $\left\{\theta_{j}\right\}_{0}^{k-1}$ of unknown inputs is considered as deterministic in the design of the following state filter

$$
\begin{aligned}
& \hat{x}_{k / k}^{\theta}=\hat{x}_{k / k-1}^{\theta}+K_{k}^{\theta}\left(y_{k}-C \hat{x}_{k / k-1}^{\theta}\right) \\
& P_{k / k}^{\theta}=\left(I-K_{k}^{\theta} C\right) P_{k / k-1}^{\theta}\left(I-K_{k}^{\theta} C\right)^{T}+K_{k}^{\theta} K_{k}^{\theta T} \\
& \hat{x}_{k+1 / k}^{\theta}=A \hat{x}_{k / k}^{\theta}+B u_{k} \\
& P_{k+1 / k}^{\theta}=A P_{k / k}^{\theta} A^{T}+W
\end{aligned}
$$

where $\hat{x}_{k / k-1}^{\theta}$ is the state prediction of covariance matrix $P_{k / k-1}^{\theta}=E\left\{\left(x_{k}-\hat{x}_{k / k-1}^{\theta}\right)\left(x_{k}-\hat{x}_{k / k-1}^{\theta}\right)^{T}\right\} \quad$ based on measurements available until time $k-1$ and $\left\{\theta_{j}\right\}_{0}^{k-1}$, where $\hat{x}_{k / k}^{\theta}$ is the state estimate of covariance matrix $P_{k / k}^{\theta}=E\left\{\left(x_{k}-\hat{x}_{k / k}^{\theta}\right)\left(x_{k}-\hat{x}_{k / k}^{\theta}\right)^{T}\right\}$ based on measurements available until time $k$ and $\left\{\theta_{j}\right\}_{0}^{k-1}$. From (1) and (2), the state prediction error $e_{k+1 / k}^{\theta}=x_{k+1}-\hat{x}_{k+1 / k}^{\theta}$ and the state estimation error $e_{k / k}^{\theta}=x_{k}-\hat{x}_{k / k}^{\theta}$ propagate as

$$
\begin{aligned}
& e_{k+1 / k}^{\theta}=A e_{k / k}^{\theta}+F d_{k}^{\theta}+w_{k} \\
& e_{k / k}^{\theta}=\left(I-K_{k}^{\theta} C\right) e_{k / k-1}^{\theta}-K_{k}^{\theta} v_{k}
\end{aligned}
$$

Under $E\left\{e_{k / k-1}^{\theta}\right\}=F d_{k-1}^{\theta}$ with $F=\left[\begin{array}{lllll}f^{1} & . . & f^{i} & . . & f^{q}\end{array}\right]$, we have $E\left\{e_{k / k}^{\theta}\right\}=\left(I-K_{k}^{\theta} C\right) E\left\{e_{k / k-1}^{\theta}\right\}=0$ (and thus $E\left\{e_{k+1 / k}^{\theta}\right\}=F d_{k}^{\theta}$ ) if and only if the state feedback gain $K_{k}^{\theta} \in \Re^{n, m}$ satisfies the unknown inputs decoupling constraint $\left(I-K_{k}^{\theta} C\right) F d_{k-1}^{\theta}=0$ in [24] relaxed under (1.d) as

$$
K_{k}^{\theta} C F_{k-1}^{\theta}=F_{k-1}^{\theta} \text { with } F_{k-1}^{\theta}=\left[\begin{array}{lllll}
\rho_{k-1}^{1} f^{1} & . . & \rho_{k-1}^{i} f^{i} & . . & \rho_{k-1}^{q} f^{q}
\end{array}\right]
$$

Under $\operatorname{rank}(C F)=\operatorname{rank}(F)=q$ the existence condition $\operatorname{rank}\left(C F_{k-1}^{\theta}\right)=\operatorname{rank}\left(F_{k-1}^{\theta}\right)=s_{k}$ with $s_{k}=\sum_{i=1}^{q} \rho_{k-1}^{i}$ for a solution to $(4)$ is satisfied $\forall \theta_{k-1}$. This paper proposes to parameterize the solution to equation (4) from constant size matrices as follows

$$
K_{k}^{\theta}=K_{k}^{0}+\mu_{k}^{\theta} G_{k}^{\theta}
$$

with $K_{k}^{0} \in \Re^{n, m}, \mu_{k}^{\theta}=\left(I-K_{k}^{0} C\right) F_{k-1}^{\theta}$ and $G_{k}^{\theta} \in \mathfrak{R}^{q, m}$. Substituting (5) in (4), we obtain

$$
\mu_{k}^{\theta} G_{k}^{\theta} C F_{k-1}^{\theta}=\mu_{k}^{\theta}
$$

With $I_{k-1}^{\theta}=\operatorname{diag}\left[\rho_{k-1}^{1} \quad \rho_{k-1}^{i} \quad \rho_{k-1}^{q}\right]$ so that $\mu_{k}^{\theta} I_{k-1}^{\theta}=\mu_{k}^{\theta}$ since $\mu_{k}^{\theta}$ has a special structure with zero columns when $d_{k-1}^{\theta}$ has 
zero components, we deduce from (6) that the state feedback gain (5) satisfies (4) if and only if $G_{k}^{\theta}$ satisfies

$$
G_{k}^{\theta} C F_{k-1}^{\theta}=I_{k-1}^{\theta}
$$

Suggested by the structure of the state feedback gain (5), let

$$
\begin{aligned}
& \hat{d}_{k-1 / k}^{\theta}=G_{k}^{\theta}\left(y_{k}-C \hat{x}_{k / k-1}^{\theta}\right) \\
& Q_{k-1 / k}^{\theta}=E\left\{\varepsilon_{k-1 / k}^{\theta} \varepsilon_{k-1 / k}^{\theta T}{ }^{T}\right\}
\end{aligned}
$$

with $\varepsilon_{k-1 / k}^{\theta}=\hat{d}_{k-1 / k}^{\theta}-d_{k-1}^{\theta}$ where $E\left\{\hat{d}_{k-1 / k}^{\theta}\right\}=G_{k}^{\theta} C E\left\{k_{k / k-1}^{\theta}\right\}=d_{k-1}^{\theta}$ under (4) and (7). The state estimator (2) and the intermittent unknown inputs estimator (8) will be designed by minimising the trace of $P_{k / k}^{\theta}$ and $Q_{k-1 / k}^{\theta}$ with respect to $K_{k}^{\theta}$ and $G_{k}^{\theta}$ under (4) and (7). The necessary and sufficient conditions so that $\lim _{k \rightarrow \infty} P_{k+1 / k}^{\theta}<\infty$ for any sequence $\left\{\theta_{j}\right\}_{0}^{b_{0}}$ will be established.

\section{KALMAN FILTER FOR STOCHASTIC LINEAR SYSTEM SUBJECT TO INTERMITTENT UNKNOWN INPUTS}

The proposed IIKF is described by theorem 3.1.

Theorem 3.1: The Unbiased Minimum Variance (UMV) state estimate $\hat{x}_{k / k}^{\theta}$ of covariance $P_{k / k}^{\theta}$ is generated by the following modified Kalman filter

$$
\begin{aligned}
& \hat{x}_{k / k}^{\theta}=\left(I-K_{k}^{0} C\right)\left(\hat{x}_{k / k-1}^{\theta}+F_{k-1}^{\theta} \hat{d}_{k-1 / k}^{\theta}\right)+K_{k}^{0} y_{k} \\
& P_{k / k}^{\theta}=\left(I-K_{k}^{0} C\right)\left(P_{k / k-1}^{\theta}+F_{k-1}^{\theta} Q_{k-1 / k}^{\theta} \theta_{k-1}^{\theta T}\right)\left(I-K_{k}^{0} C\right)^{T}+K_{k}^{0} K_{k}^{0 T} \\
& \hat{x}_{k+1 / k}^{\theta}=A \hat{x}_{k / k}^{\theta}+B u_{k} \quad P_{k+1 / k}^{\theta}=A P_{k / k}^{\theta} A^{T}+W
\end{aligned}
$$

with $K_{k}^{0}=P_{k / k-1}^{\theta} C\left(C P_{k / k-1}^{\theta} C^{T}+I\right)^{-1}$, updated online from the additive quantities $F_{k-1}^{\theta} \hat{d}_{k-1 / k}^{\theta}$ and $F_{k-1}^{\theta} Q_{k-1 / k}^{\theta} F_{k-1}^{\theta T}$ depending on the unknown inputs estimate $\hat{d}_{k-1 / k}^{\theta}$ of covariance $Q_{k-1 / k}^{\theta}$ given by

$$
\begin{aligned}
& \hat{d}_{k-1 / k}^{\theta}=G_{k}^{\theta}\left(y_{k}-C \hat{x}_{k / k-1}^{\theta}\right) \\
& Q_{k-1 / k}^{\theta}=\left[\left(C F_{k-1}^{\theta}\right)^{T}\left(C P_{k / k-1}^{\theta} C^{T}+I\right)^{-1} C F_{k-1}^{\theta}\right]^{+}
\end{aligned}
$$

with $G_{k}^{\theta}=Q_{k-1 / k}^{\theta}\left(C F_{k-1}^{\theta}\right)^{T}\left(C P_{k / k-1}^{\theta} C^{T}+I\right)^{-1}$. The ith component $\hat{d}_{k-1 / k}^{\theta i}$ of $\hat{d}_{k-1 / k}^{\theta}$ represents the estimate of $\rho_{k-1}^{i} d_{k-1}^{i}$ (with $\hat{d}_{k-1 / k}^{\theta i}=0$ when $\left.\rho_{k-1}^{i}=0\right)$ and the ith component $Q_{k-1 / k}^{\theta i}$ on the diagonal part of $Q_{k-1 / k}^{\theta}$ represents the variance of $\hat{d}_{k-1 / k}^{\theta i}$ (with $Q_{k-1 / k}^{\theta i}=0$ when $\left.\rho_{k-1}^{i}=0\right)$. The IIKF is initialized by $\hat{x}_{0 /-1}^{\theta}=\bar{x}_{0}, P_{0 /-1}^{\theta}=P_{0} \geq 0$ and $\theta_{-1}=\{0, . ., 0, . ., 0\}$.

Proof. Let $\quad x_{k}^{\theta}=\left[\begin{array}{ll}x_{k}^{T} & d_{k-1}^{\theta T}\end{array}\right]^{T}, \quad \hat{X}_{k / k}^{\theta}=\left[\begin{array}{cc}x_{k / k}^{\theta T} & \hat{d}_{k-1 / k}^{\theta T}\end{array}\right]^{T}$, $\Omega_{k / k}^{\theta}=E\left\{\left[\begin{array}{c}e_{k / k}^{\theta} \\ \varepsilon_{k-1 / k}^{\theta}\end{array}\right]\left[\begin{array}{c}e_{k / k}^{\theta} \\ \varepsilon_{k-1 / k}^{\theta}\end{array}\right]^{T}\right\} \quad$ and $\quad L_{k}^{\theta}=\left[\begin{array}{ll}K_{k}^{\theta T} & G_{k}^{\theta T}\end{array}\right]^{T}$. The state estimator (2) and the unknown inputs estimator (8) can then be jointly expressed

$$
\begin{aligned}
& \hat{X}_{k / k}^{\theta}=\left[\begin{array}{l}
I \\
0
\end{array}\right] \hat{x}_{k / k-1}^{\theta}+L_{k}^{\theta}\left(y_{k}-C \hat{x}_{k / k-1}^{\theta}\right) \\
& \left.\left.\Omega_{k / k}^{\theta}=\left[\begin{array}{l}
I \\
0
\end{array}\right]-L_{k}^{\theta} C\right) P_{k / k-1}^{\theta}\left[\begin{array}{l}
I \\
0
\end{array}\right]-L_{k}^{\theta} C\right)^{T}+L_{k}^{\theta} L_{k}^{\theta^{T}} \\
& \hat{x}_{k+1 / k}^{\theta}=\left[\begin{array}{lll}
A & 0
\end{array}\right] \hat{X}_{k / k}^{\theta}+B u_{k} \quad P_{k+1 / k}^{\theta}=\left[\begin{array}{lll}
A & 0
\end{array}\right] \Omega_{k / k}^{\theta}\left[\begin{array}{ll}
A & 0
\end{array}\right]^{T}+W
\end{aligned}
$$

Under $\operatorname{tr}\left(P_{k / k-1}^{\theta}\right)$ minimum, $\hat{X}_{k / k}^{\theta}$ is the UMV estimate of $X_{k}^{\theta}$ (and thus $\operatorname{tr}\left(P_{k+1 / k}^{\theta}\right)$ minimum) if and only if the augmented gain $L_{k}^{\theta}=\left[\begin{array}{ll}K_{k}^{\theta T} & G_{k}^{\theta T}\end{array}\right]^{T}$ is solution to the constrained optimization problem

$$
\min _{L_{k}^{\theta} t r\left(\Omega_{k / k}^{\theta}\right)}^{\theta} \text { s.t. } L_{k}^{\theta} C F_{k-1}^{\theta}=\left[\begin{array}{c}
F_{k-1}^{\theta} \\
I_{k-1}^{\theta}
\end{array}\right]
$$

The solution to (13) is difficult to obtain since $K_{k}^{\theta}$ depends on $\quad G_{k}^{\theta} \quad$ from $\quad K_{k}^{\theta}=K_{k}^{0}+\mu_{k}^{\theta} G_{k}^{\theta}$. Let $\quad \bar{X}_{k}^{\theta}=T_{k} X_{k}^{\theta}, \quad \hat{\bar{X}}_{k / k}^{\theta}=T_{k} \hat{X}_{k / k}^{\theta}$, $\bar{\Omega}_{k / k}^{\theta}=T_{k} \Omega_{k / k}^{\theta} T_{k}^{T}$ and $\bar{L}_{k}^{\theta}=T_{k} L_{k}^{\theta}$ where $T_{k}$ is an arbitrary nonsingular transformation matrix of appropriate dimension. The filter (12) can then be equivalently rewritten

$$
\begin{aligned}
& \hat{\bar{X}}_{k / k}^{\theta}=T_{k}\left[\begin{array}{l}
I \\
0
\end{array}\right] \hat{x}_{k / k-1}^{\theta}+\bar{L}_{k}^{\theta}\left(y_{k}-C \hat{x}_{k / k-1}^{\theta}\right) \\
& \bar{\Omega}_{k / k}^{\theta}=\left(T_{k}\left[\begin{array}{l}
I \\
0
\end{array}\right]-\bar{L}_{k}^{\theta} C\right) P_{k / k-1}^{\theta}\left(T_{k}\left[\begin{array}{l}
I \\
0
\end{array}\right]-\bar{L}_{k}^{\theta} C\right)^{T}+\bar{L}_{k}^{\theta} \bar{L}_{k}^{\theta^{T}} \\
& \hat{x}_{k+1 / k}^{\theta}=\left[\begin{array}{ll}
A & 0
\end{array}\right] T_{k}^{-1} \hat{\bar{X}}_{k / k}^{\theta}+B u_{k} \\
& P_{k+1 / k}^{\theta}=\left[\begin{array}{ll}
A & 0
\end{array}\right] T_{k}^{-1} \bar{\Omega}_{k / k}^{\theta} T_{k}^{-T}\left[\begin{array}{ll}
A & 0
\end{array}\right]^{T}+W
\end{aligned}
$$

Under $\operatorname{tr}\left(P_{k / k-1}^{\theta}\right)$ minimum, $\hat{\bar{X}}_{k / k}^{\theta}$ is the UMV estimate of $\bar{X}_{k}^{\theta}$ (and thus $\operatorname{tr}\left(P_{k+1 / k}^{\theta}\right)$ minimum) if and only if the transformed gain $\bar{L}_{k}^{\theta}$ is solution to

$$
\min _{\bar{L}_{k}^{\theta} \operatorname{tr}\left(\bar{\Omega}_{k / k}^{\theta}\right)}^{\theta} \text { s.t. } \bar{L}_{k}^{\theta} C F_{k-1}^{\theta}=T_{k}\left[\begin{array}{c}
F_{k-1}^{\theta} \\
I_{k-1}^{\theta}
\end{array}\right]
$$

With $T_{k}=\left[\begin{array}{cc}I & -\mu_{k}^{\theta} \\ 0 & I\end{array}\right]$ so that $\bar{L}_{k}^{\theta}=T_{k}\left[\begin{array}{ll}K_{k}^{\theta T} & G_{k}^{\theta T}\end{array}\right]^{T}=\left[\begin{array}{ll}K_{k}^{0 T} & G_{k}^{\theta T}\end{array}\right]^{T}$ where $K_{k}^{0}$ is now decoupled from $G_{k}^{\theta}$, we can verify that the transformed algebraic constraints in (15) reduce to $G_{k}^{\theta} C F_{k-1}^{\theta}=I_{k-1}^{\theta}$. So, after straightforward manipulations, (15) becomes

$$
\left[\begin{array}{c}
K_{k}^{0} \\
G_{k}^{\theta}
\end{array}\right]
$$

with $\bar{\Omega}_{k / k}^{\theta}=\left[\begin{array}{cc}P_{k / k}^{0} & \left(P_{k / k-1}^{\theta} C^{T}-K_{k}^{0} H_{k}\right) G_{k}^{\theta T} \\ G_{k}^{\theta}\left(P_{k / k-1}^{\theta} C^{T}-K_{k}^{0} H_{k}\right)^{T} & Q_{k-1 / k}^{\theta}\end{array}\right]$

where $P_{k / k}^{0}=\left(I-K_{k}^{0} C\right) P_{k / k-1}^{\theta}\left(I-K_{k}^{0} C\right)^{T}+K_{k}^{0} K_{k}^{0 T}, Q_{k-1 / k}^{\theta}=G_{k}^{\theta} H_{k} G_{k}^{\theta T}$ and $H_{k}=C P_{k / k-1}^{\theta} C^{T}+I$. With $\operatorname{tr}\left(\bar{\Omega}_{k / k}^{\theta}\right)=\operatorname{tr}\left(P_{k / k}^{0}\right)+\operatorname{tr}\left(Q_{k-1 / k}^{\theta}\right)$ obtained from (17), we deduce that the global solution to (16) corresponds to the local solutions of the following decoupled optimisation problems

$$
\min _{k}^{0} \operatorname{tr}\left(P_{k / k}^{0}\right)
$$

and

$$
\min _{G_{k}^{\theta}} \operatorname{tr}\left(Q_{k-1 / k}^{\theta}\right) \text { s.t. } G_{k}^{\theta} C F_{k-1}^{\theta i}=I_{k-1}^{\theta i} \text { for } i=1, . ., q
$$

where $F_{k-1}^{\theta i}$ and $I_{k-1}^{\theta i}$ represent the ith column of $F_{k-1}^{\theta}$ and $I_{k-1}^{\theta}$.

The unique solution to (18.a) coincides with the Kalman filter's gain $K_{k}^{0}=P_{k / k-1}^{\theta} C\left(C P_{k / k-1}^{\theta} C^{T}+I\right)^{-1}$. The existence of the ith 
constraint in (18.b) is conditioned by $\rho_{k-1}^{i}=1$ and the solution to (18.b) can be derived by minimising

$$
\Phi_{k}^{\theta}=0.5 \operatorname{tr}\left(G_{k}^{\theta} H_{k} G_{k}^{\theta T}\right)+\sum_{i=1}^{q} \lambda_{k}^{\theta i T}\left(G_{k}^{\theta} C F_{k-1}^{\theta i}-I_{k-1}^{\theta i}\right)
$$

where $\lambda_{k}^{\theta i} \in \mathfrak{R}^{q, 1}$ is the Lagrange parameters vector satisfying $\lambda_{k}^{\theta i}=0$ when $\rho_{k-1}^{i}=0$ and $\lambda_{k}^{\theta i} \neq 0$ when $\rho_{k-1}^{i}=1$. The optimality conditions of $\Phi_{k}^{\theta}$ gives

$$
\begin{aligned}
& \frac{\partial \Phi_{k}^{\theta}}{\partial G_{k}^{\theta}}=G_{k}^{\theta} H_{k}+\sum_{i=1}^{q} \lambda_{k}^{\theta i}\left(C F_{k-1}^{\theta i}\right)^{T}=0 \\
& \frac{\partial \Phi_{k}^{\theta}}{\partial \lambda_{k}^{i}}=G_{k}^{\theta} C F_{k-1}^{\theta i}-I_{k-1}^{\theta i}=0 \text { if } \rho_{k-1}^{i}=1 \text { for } i=1, . ., q
\end{aligned}
$$

The solution $G_{k}^{\theta}=-\left\{\sum_{i=1}^{q} \lambda_{k}^{\theta i}\left(C F_{k-1}^{\theta i}\right)^{T}\right\} H_{k}^{-1}$ to (20.a) substituted in (20.b) gives

$$
-\lambda_{k}^{\theta}\left(C F_{k-1}^{\theta}\right)^{T} H_{k}^{-1} C F_{k-1}^{\theta}=I_{k-1}^{\theta}
$$

where $\lambda_{k}^{\theta}=\left[\begin{array}{lll}\lambda_{k}^{\theta 1} & \lambda_{k}^{\theta i} & \lambda_{k}^{\theta_{q}}\end{array}\right] \in \Re^{q, q}$ represents the Lagrange parameters matrix. The solution to (21), expressed $\lambda_{k}^{\theta}=-I_{k-1}^{\theta}\left[\left(C F_{k-1}^{\theta}\right)^{T} H_{k}^{-1} C F_{k-1}^{\theta}\right]^{+}=\left[\left(C F_{k-1}^{\theta}\right)^{T} H_{k}^{-1} C F_{k-1}^{\theta}\right]^{+}$from $F_{k-1}^{\theta} I_{k-1}^{\theta}=F_{k-1}^{\theta}$, substituted in $G_{k}^{\theta}=-\lambda_{k}^{\theta}\left(C F_{k-1}^{\theta}\right)^{T} H_{k}^{-1}$ gives

$$
G_{k}^{\theta}=\left[\left(C F_{k-1}^{\theta}\right)^{T} H_{k}^{-1} C F_{k-1}^{\theta}\right]^{+}\left(C F_{k-1}^{\theta}\right)^{T} H_{k}^{-1}
$$

leading to $Q_{k-1 / k}^{\theta}=G_{k}^{\theta} H_{k} G_{k}^{\theta T}=\left[\left(C F_{k-1}^{\theta}\right)^{T} H_{k}^{-1} C F_{k-1}^{\theta}\right]^{+}$via $X^{+} X X^{+}=X^{+}$ ( $x^{+}$is the unique Moore-Penrose generalized inverse of $X$ ).

After some manipulations, the IIKF of theorem 3.1 is recovered by substituting the inverse transformation $T_{k}^{-1}=\left[\begin{array}{cc}I & \mu_{k}^{\theta} \\ 0 & I\end{array}\right]$ in the optimized filter (14). Its optimality is ensured by the invariance property to linear transforms of optimal linear filters. The IIKF must be initialized with $\theta_{-1}=\{0, \ldots, \ldots, ., 0\}$ since $\bar{x}_{0}$ represents the unbiased prediction of the initial state $x_{0}$.

\section{End of the proof}

From (9.b) and (10), we derive the IIKF's Riccati Difference Equation (RDE)

$$
P_{k+1 / k}^{\theta}=\left(A-K_{k}^{p} C\right)\left(P_{k / k-1}^{\theta}+F_{k-1}^{\theta} Q_{k-1 / k}^{\theta} F_{k-1}^{\theta T}\right)\left(A-K_{k}^{p} C\right)^{T}+K_{k}^{p} K_{k}^{p T}+W
$$

with $K_{k}^{p}=A K_{k}^{0}=A P_{k / k-1}^{\theta} C^{T}\left(C P_{k / k-1}^{\theta} C^{T}+I\right)^{-1}$.

Theorem 3.2: Under the necessary and sufficient conditions

$$
\begin{aligned}
& \operatorname{rank}\left[\begin{array}{cc}
-I z+A & F \\
C & 0
\end{array}\right]=n+q, \forall|z| \geq 1 \\
& \operatorname{rank}\left[-I e^{j w}+A \quad W^{1 / 2}\right]=n, \forall w \in[0,2 \pi]
\end{aligned}
$$

we have $\lim _{k \rightarrow \infty} P_{k+1 / k}^{\theta}<\infty$ for any arrival sequence $\left\{\theta_{j}\right\}_{0}^{\infty}$ of unknown inputs.

Proof. Under persistent unknown inputs i.e. $\theta_{k}=\{1, . ., 1, ., 1\}$ $\forall k \geq 0$, we have $d_{k}^{\theta}=\left[\begin{array}{lllll}d_{k}^{1} & . & d_{k}^{i} & . . & d_{k}^{q}\end{array}\right]^{T} \forall k \geq 0$ or $F_{k}^{\theta}=F \quad \forall k \geq 0$ and (23) gives

$$
\widehat{P}_{k+1 / k}=\left(A-\widehat{K}_{k}^{p} C\right)\left(\widehat{P}_{k / k-1}+F \hat{Q}_{k-1 / k} F^{T}\right)\left(A-\widehat{K}_{k}^{p} C\right)^{T}+\widehat{K}_{k}^{p} \widehat{K}_{k}^{p T}+W
$$

with $\hat{K}_{k}^{p}=A \hat{P}_{k / k-1} C^{T} \hat{H}_{k}^{-1}, \quad \hat{Q}_{k-1 / k}=\left[(C F)^{T} \hat{H}_{k}^{-1} C F\right]^{-1}, \hat{H}_{k}=C \widehat{P}_{k / k-1} C^{T}+I$ and $\hat{P}_{0 /-1}=P_{0} \geq 0$. The RDE (25) can be rewritten
$\hat{P}_{k+1 / k}=\left(A-\widehat{K}_{k} C\right) \hat{P}_{k / k-1}\left(A-\widehat{K}_{k} C\right)^{T}+\widehat{K}_{k} \hat{K}_{k}^{T}+W$

with $\quad \hat{K}_{k}=A\left(\hat{K}_{k}^{0}+\left(I-\widehat{K}_{k}^{0} C\right) F \widehat{G}_{k}\right), \quad \hat{K}_{k}^{0}=\hat{P}_{k / k-1} C^{T}\left(C \hat{P}_{k / k-1} C^{T}+I\right)^{-1} \quad$ and $\hat{G}_{k}=\left[(C F)^{T} \hat{H}_{k}^{-1} C F\right]^{-1}(C F)^{T} \hat{H}_{k}^{-1}$.

The appendix shows that the RDE (26) coincides with the time-invariant UIKF's RDE in [7] described by

$$
P_{k+1 / k}=\left(A-K_{k} C\right) P_{k / k-1}\left(A-K_{k} C\right)^{T}+K_{k} K_{k}^{T}+W
$$

with $\quad K_{k}=A\left(\bar{K}_{k} \Sigma+F \Pi\right), \quad \bar{K}_{k}=N P_{k / k-1} \bar{C}^{T}\left(\bar{C} P_{k / k-1} \bar{C}^{T}+\Sigma \Sigma^{T}\right)^{-1} \in \Re^{n, m-q}$, $\Pi=(C F)^{+}, \quad N=I-F \tilde{C}, \quad \tilde{C}=\Pi C, \quad \bar{C}=\Sigma C, \quad \Sigma=\beta(I-C F \Pi) \quad$ and $\beta \in \Re^{m-q, m}$ so that $\operatorname{rank}(\Sigma)=m-q$ with $q=\operatorname{rank}(C F)$. This result also shows that the IIKF's RDE (23) is equivalent to a timevarying structure version of (27) with $K_{k}=A\left(\bar{K}_{k}^{\theta} \Sigma_{k-1}^{\theta}+F_{k-1}^{\theta} \Pi_{k-1}^{\theta}\right)$, $\bar{K}_{k}^{\theta}=N_{k}^{\theta} P_{k / k-1} \bar{C}_{k}^{\theta T}\left(\bar{C}_{k}^{\theta} P_{k / k-1} \bar{C}_{k}^{\theta T}+\Sigma_{k}^{\theta} \Sigma_{k}^{\theta T}\right)^{-1}, \Pi_{k}^{\theta}=\left(C F_{k-1}^{\theta}\right)^{+}, N_{k}^{\theta}=I-F_{k-1}^{\theta} \widetilde{C}_{k}^{\theta}$ $\widetilde{C}_{k}^{\theta}=\Pi_{k}^{\theta} C, \bar{C}_{k}^{\theta}=\Sigma_{k}^{\theta} C, \Sigma_{k}^{\theta}=\beta_{k}^{\theta}\left(I-C F_{k-1}^{\theta} \Pi_{k}^{\theta}\right)$ and $\beta_{k}^{\theta} \in \mathfrak{R}^{m-s_{k}, m}$ so that $\operatorname{rank}\left(\Sigma_{k}^{\theta}\right)=m-s_{k}$ where the variable size of the gain $\bar{K}_{k}^{\theta} \in \mathfrak{R}^{n, m-s_{k}}$ depends on $s_{k}=\sum_{i=1}^{q} \rho_{k-1}^{i}$ (contrary to the time-varying RDE, but fixed structure in [7], defined with $\left.s_{k}=q, \forall k\right)$. The degrees of freedom $\bar{K}_{k}^{\theta} \in \Re^{n, m-s_{k}}$ minimizing $\operatorname{tr}\left(P_{k+1 / k}^{\theta}\right)$ are always greater or equal to the degrees of freedom $\bar{K}_{k} \in \mathfrak{R}^{n, m-q}$ minimizing $\operatorname{tr}\left(P_{k+1 / k}\right) \quad$ since $s_{k} \leq q$. Without further mathematical development, this reasoning leads to $P_{k+1 / k}^{\theta} \leq P_{k+1 / k}$ for any sequence $\left\{\theta_{j}\right\}_{0}^{k-1}$. Under $P_{0 /-1}=P_{0} \geq 0$, the necessary and sufficient existence conditions (24) for an unique stabilising solution $P \geq 0$ to the following Algebraic Riccati Difference Equation (ARDE)

$$
P=(A-K C) P(A-K C)^{T}+K K^{T}+W
$$

associated to the time-invariant $\mathrm{RDE}$ (27) (all the modes of $A-K C$ inside the unique circle) have been established in [7]. Under (24), we conclude that $\lim _{k \rightarrow \infty} P_{k+1 / k}^{\theta} \leq P<\infty$ for any sequence $\left\{\theta_{j}\right\}_{0}^{\infty}$.

End of the proof

When the arrival sequence of each component of the unknown inputs vector follows a Bernoulli process with $\lambda=\operatorname{Pr}\left[\rho_{k}^{i}=1\right] \in\left[\begin{array}{ll}0 & 1\end{array}\right]$, the rank relations (24) ensure that there exists no critical value for the unknown inputs occurrence rate $\lambda$ above which the estimation error covariance becomes unbounded.

\section{CONCLUSION}

In this paper a Kalman Filter for discrete-time stochastic linear systems subject to intermittent unknown inputs has been presented. The decoupling constraint linked to the binary sequence describing the arrival of unknown inputs has been parameterized from constant size matrices in order to allow a two-stage optimisation strategy. The obtained filter has been presented as a standard Kalman filter updated online from the 
intermittent unknown inputs estimation. When the arrival sequences of unknown inputs follow binary state Markov chains or Bernoulli processes, the study of less conservative stochastic stability conditions is currently under consideration by the authors.

Appendix: The RDE (26) coincides with the standard UIKF's RDE (27) i.e. $\widehat{K}_{k}=K_{k}$ when $\hat{P}_{k / k-1}=P_{k / k-1}$ : Define the non singular output transformation matrix

$$
\hat{Z}_{k}=\left[\begin{array}{cc}
I & 0 \\
-\widehat{J}_{k} & I
\end{array}\right]\left[\begin{array}{l}
\Sigma \\
\Pi
\end{array}\right] \in \mathfrak{R}^{m, m}
$$

with $\widehat{J}_{k}=\widetilde{C} \widehat{C}_{k / k-1} \bar{C}^{T} \overline{\bar{H}}_{k}^{-1}$ and $\overline{\bar{H}}_{k}=\bar{C} \widehat{P}_{k / k-1} \bar{C}^{T}+\Sigma \Sigma^{T} \quad$ ( $\hat{J}_{k}$ is called the adaptive noise canceller gain in [21]) so that

$$
\hat{Y}_{k}=\hat{Z}_{k}\left(C \hat{P}_{k / k-1} C^{T}+I\right) \hat{Z}_{k}^{T}=\left[\begin{array}{cc}
\hat{\bar{H}}_{k} & 0 \\
0 & \widetilde{\tilde{H}}_{k}
\end{array}\right]
$$

with $\widetilde{\widetilde{H}}_{k}=\widetilde{C} \widehat{S}_{k / k} \widetilde{C}^{T}+H H^{T}$ and $\hat{S}_{k / k}=\hat{P}_{k / k-1}-\hat{P}_{k / k-1} \bar{C}^{T} \overline{\bar{H}}_{k}^{-1} \bar{C} \hat{P}_{k / k-1}$. The constrained gain $\hat{G}_{k}=\left[(C F)^{T} \hat{H}_{k}^{-1} C F\right]^{-1}(C F)^{T} \hat{H}_{k}^{-1}$ can be equivalently expressed

$$
\begin{aligned}
& \hat{G}_{k}=\left(\left(\hat{Z}_{k} C F\right)^{T} \hat{Y}_{k}^{-1} \hat{Z}_{k} C F\right)^{-1}\left(\hat{Z}_{k} C F\right)^{T} \hat{Y}_{k}^{-1} \hat{Z}_{k} \\
& \left.\left.=\left(\left[\begin{array}{ll}
0 & I
\end{array}\right]\left[\begin{array}{cc}
\overline{\bar{H}}_{k} & 0 \\
0 & \widetilde{\widetilde{H}}_{k}
\end{array}\right]\right)^{-1}\left[\begin{array}{l}
0 \\
I
\end{array}\right]\right)^{-1}\left[\begin{array}{cc}
0 & I
\end{array}\right] \begin{array}{cc}
\overline{\bar{H}}_{k} & 0 \\
0 & \widetilde{\tilde{H}}_{k}
\end{array}\right]^{-1} \hat{Z}_{k}=\Pi-\widehat{J}_{k} \Sigma
\end{aligned}
$$

By the same way, the free gain $\hat{K}_{k}^{0}=\hat{P}_{k / k-1} C^{T}\left(C \hat{P}_{k / k-1} C^{T}+I\right)^{-1}$ can be equivalently expressed

$$
\begin{aligned}
& \hat{K}_{k}^{0}=\hat{P}_{k / k-1} C^{T} \hat{Z}_{k}^{T} \hat{Y}_{k}^{-1} \hat{Z}_{k} \\
& =\hat{P}_{k / k-1}\left[\begin{array}{ll}
\bar{C}^{T} \hat{\bar{H}}_{k}^{-1} & \left(\widetilde{C}^{T}-\bar{C}^{T} \hat{J}_{k}^{T}\right) \hat{\widetilde{H}}_{k}^{-1}
\end{array}\right]\left[\begin{array}{c}
\Sigma \\
\Pi-\widehat{J}_{k} \Sigma
\end{array}\right]
\end{aligned}
$$

The gain $\hat{K}_{k}=A\left(\hat{K}_{k}^{0}+\left(I-\hat{K}_{k}^{0} C\right) F \widehat{G}_{k}\right)$ is rewritten $\widehat{K}_{k}=A \widehat{K}_{k}^{0}\left(I-C F\left(H-\widehat{J}_{k} \Sigma\right)\right)+A F\left(H-\widehat{J}_{k} \Sigma\right)$ and from (A.3) and (A.4), we derive the following expression

$$
\begin{aligned}
& \hat{K}_{k}=A \widehat{P}_{k / k-1}\left[\begin{array}{ll}
\bar{C}^{T} \overline{\bar{H}}_{k}^{-1} & \left(\widetilde{C}^{T}-\bar{C}^{T} \hat{J}_{k}^{T}\right) \tilde{\tilde{H}}_{k}^{-1}
\end{array}\right]\left[\begin{array}{c}
\Sigma \\
H-\widehat{J}_{k} \Sigma
\end{array}\right]\left(I-C F\left(\Pi-\widehat{J}_{k} \Sigma\right)\right) \\
& +A F\left(\Pi-\widehat{J}_{k} \Sigma\right)
\end{aligned}
$$

From $\Sigma C F=0$ and $\Pi C F=I$, we have $\Sigma\left[I-C F\left(\Pi-\widehat{J}_{k} \Sigma\right)\right]=\Sigma$ and

$$
\begin{aligned}
& \left(H-\widehat{J}_{k} \Sigma\right)\left[I-C F\left(\Pi-\widehat{J}_{k} \Sigma\right)\right]=\left(H-\widehat{J}_{k} \Sigma\right)-\left(H-\widehat{J}_{k} \Sigma\right) C F\left(\Pi-\widehat{J}_{k} \Sigma\right) \\
& =\left(H-\widehat{J}_{k} \Sigma\right)-\left(\Pi-\widehat{J}_{k} \Sigma\right)=0
\end{aligned}
$$

leading to

$$
\begin{aligned}
& \hat{K}_{k}=A \hat{P}_{k / k-1}\left[\begin{array}{ll}
\left.\bar{C}^{T} \hat{\bar{H}}_{k}^{-1}\left(\tilde{C}^{T}-\bar{C}^{T} \hat{J}_{k}^{T}\right) \hat{\tilde{H}}_{k}^{-1}\right]
\end{array}\right]\left[\begin{array}{l}
\Sigma \\
0
\end{array}\right]+A F\left(\Pi-\hat{J}_{k} \Sigma\right)
\end{aligned}
$$

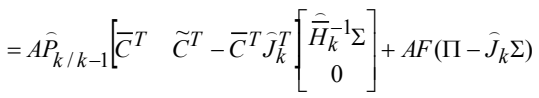

$$
\begin{aligned}
& =A \widehat{P}_{k / k-1} \bar{C}^{T} \overline{\bar{H}}_{k}^{-1} \Sigma+A F\left(\Pi-\widehat{J}_{k} \Sigma\right) \\
& =A\left(\widehat{P}_{k / k-1} \bar{C}^{T} \overline{\bar{H}}_{k}^{-1}-F \widehat{J}_{k}\right) \Sigma+A F \Pi \\
& =A\left(\widehat{P}_{k / k-1} \bar{C}^{T} \overline{\bar{H}}_{k}^{-1}-F \widetilde{C} \widehat{P}_{k / k-1} \bar{C}^{T} \overline{\bar{H}}_{k}^{-1}\right) \Sigma+A F \Pi \\
& =A(I-F \widetilde{C}) \widehat{P}_{k / k-1} \bar{C}^{T} \hat{\bar{H}}_{k}^{-1} \Sigma+A F \Pi=A N \widehat{P}_{k / k-1} \bar{C}^{T} \overline{\bar{H}}_{k}^{-1} \Sigma+A F \Pi \\
& =A\left(\bar{K}_{k} \Sigma+F \Pi\right)=K_{k}
\end{aligned}
$$

We conclude that $\hat{K}_{k}=K_{k}$ when $\hat{P}_{k / k-1}=P_{k / k-1}$ and thus $\hat{P}_{k / k-1}=P_{k / k-1} \forall k \geq 0$ when $\hat{P}_{0 /-1}=P_{0 /-1}$.

\section{ACKNOWLEDGMENT}

The authors thank the anonymous reviewers for the constructive comments to improve the manuscript.

\section{REFERENCES}

[1] A.T. Alouani, T.R. Rice, and W.D. Blair, "A two-stage filter for state estimation in the presence of dynamic stochastic bias," in Proc. of the American Control Conference, Chicago, pp. 1784-1788, 1992.

[2] M. Blanke, M. Kinnaert, J. Lunze, and M. Staroswieki, Diagnosis and Fault Tolerant Control, Springer, Berlin, 2006.

[3] A. Censi, "On the performance of Kalman filtering with intermittent observations: A geometric approach with fractals," in Proc. of the American Control Conference, St Louis, pp. 3806-3812, 2009.

[4] J. Chen, and R.J. Patton, "Optimal filtering and robust fault diagnosis of stochastic systems with unknown disturbances," Control Theory and Applications, vol. 143, no. 1, pp. 31-36, 1996.

[5] J. Chen, and R.J. Patton, Robust Model based fault diagnosis for dynamic systems, Kluwer Academic Publishers, 1999.

[6] M. Darouach, M. Zasadzinski, and J.Y. Keller, "State estimation for discrete systems with unknown inputs using state estimation of singular systems," in Proc. of the American Control Conference, Chicago, pp. 3014-3015, 1992.

[7] M. Darouach, and M. Zasadzinski, "Unbiased minimum variance estimation for systems with unknown exogenous inputs," Automatica, vol. 33, no. 4, pp. 717-719, 1997.

[8] H. Fang, Y. Shi, and J. Yi, "On stable simultaneous input and state estimation for discrete-time linear systems," International Journal of Adaptive Control and Signal Processing, Early view, Online version of record published before inclusion in an issue, 2011.

[9] A. Fletcher, S. Rangan, and V. Goyal, "Estimation from lossy sensor data: jump linear modeling and Kalman filtering," in Proc. of the International Symposium on Information Processing in Sensor Networks, California, pp. 251-258, 2004.

[10] B. Friedland, "Treatment of bias in recursive filtering," IEEE Trans. Autom. Control, vol. 14, no. 4, pp. 359-367, 1969.

[11] S. Gillijns, and B. De Moor, "Unbiased minimum-variance input and state estimation for linear discrete-time systems with direct feedthrough," Automatica, vol. 43, no.5, pp. 934-937, 2007.

[12] F.B. Hmida, K. Khémiri, J. Ragot, and M. Gossa, "Robust filtering for state and fault estimation of linear stochastic systems with unknown disturbance," Mathematical Problems in Engineering, ID 591639, 2010.

[13] M. Hou, and P.C. Müller, "Disturbance decoupled observer design: A unified viewpoint," IEEE Trans. Autom. Control, vol. 39, no. 6, pp. 1338-1341, 1994.

[14] M. Hou, and R.J. Patton, "Input observability and input reconstruction," Automatica, vol. 34, no. 6, pp. 789-794, 1998.

[15] M. Hou, and R.J. Patton, "Optimal filtering for systems with unknown inputs," IEEE Trans. Autom. Control, vol. 43, no. 3, pp. 445-449, 1998.

[16] J.P. Hespanha, P. Naghshtabrizi, and Y. Xu, "Survey of recent results in networked control systems," Proceeding of the IEEE, Vol. 95, no. 1, pp. 138-162, 2007.

[17] C.S. Hsieh, and F.C. Chen, "Optimal solution of the two stage Kalman estimator," IEEE Trans. Autom. Control, vol. 44, no. 1, pp. 194-199, 1999.

[18] M. Ignagni, "Optimal and suboptimal separate-bias Kalman filter estimators of a stochastic bias," IEEE Trans. Autom. Control, vol. 45, no. 3, pp. 547-551, 2000.

[19] T. Kailath, A. Sayed, and B. Hassibi, Linear Estimation, Prentice-Hall: Upper Saddle River, 2000.

[20] J.Y. Keller, and M. Darouach, "Optimal two-stage Kalman filter in the presence of random bias," Automatica, vol. 33, no. 9, pp. 1745-1748, 1997.

[21] J.Y. Keller, and D. Sauter, "Restricted diagonal detection filter and updating strategy for multiple fault detection and isolation," Adaptive Control and Signal Processing, vol. 25, no. 1, pp. 68-87, 2011.

[22] Y. Kim, and J. Park, "Noise response of detection filters: relation between detection space and completion space," Control Theory and Applications, vol. 150, no. 4, pp. 443-447, 2003. 
[23] K.H. Kim, J.G. Lee, and C.G. Park, "Adaptive two-stage Kalman filter in the presence of unknown random bias," Adaptive Control and Signal Processing, vol. 20, no. 6, pp. 305-319, 2006.

[24] P.K. Kitanidis, "Unbiased minimum-variance linear state estimation," Automatica, vol. 23, no 6, pp. 775-778, 1987.

[25] Y. Liang, L. Zhang, D. Zhou, and Q. Pan, "Estimation of systems with statistically-constrained inputs," Applied Mathematics and Computation, vol. 217, no. 6, pp. 2644-2656, 2010.

[26] J. Park, and G. Rizzoni, "A new interpretation of the fault detection filter," International Journal of Control, vol. 60, no. 6, pp. 1339-1351, 1994.

[27] L. Schenato, B. Sinopoli, M. Franceschetti, K. Poolla, and S. Sastry,“ Foundations of control and estimation over lossy networks," Proceedings of IEEE, vol. 95, no. 1, pp. 163-187, 2007.

[28] L. Shi, L. Xie, and R.M. Murray, "Kalman filtering over a packeddelaying network: A probabilistic approach," Automatica, vol. 45, no. 9, pp. 2134-2140, 2009.

[29] D. Simon, Optimal State Estimation: Kalman, $\mathrm{H} \infty$, and Nonlinear Approaches, Wiley-Interscience: New York, 2006.

[30] B. Sinopoli, L. Schenato, M. Franceschetti, K. Poolla, M. Jordan, and S. Sastry, "Kalman filtering with intermittent observations," IEEE Trans. Autom. Control, vol. 49, no. 9, pp. 1453-1464, 2004.

[31] A. Censi, "Kalman Filtering with intermittent observations: Convergence for semi-Markov chains and an intrinsic performance measure," IEEE Trans. Autom. Control, vol. 56, no. 2, pp. 376-381, 2011.

J.Y. Keller was born in France, Thionville, in 1964. He joined the Centre de Recherche en Automatique de Nancy (CRAN) in 1988. He received his Ph.D. degree in Automatic Control from the University of Nancy I, France, in 1991. Since 1993, he teaches as "Maître de Conférences HC" at the University Institute of Technology Henri Poincaré (IUT de Longwy). He obtained his "Habilitation à diriger des recherches" from University Henri Poincaré, Nancy, in 2003. His main interests include state filtering, fault diagnosis and fault tolerant control for large scale network controlled systems.

D. Sauter received the Doctorat ès Sciences Degree (1991) from the University Henri Poincaré, Nancy1, France. Since 1993 he is a full Professor at University of Lorraine. He has been the head of the Electrical Engineering Department during 4 years and Vice-Dean of the Faculty of Sciences and Technology. He also works with Research Center In Automatic Control of Nancy (CRAN) associated to the French National Center For Scientific Research (CNRS). His current research interests are focused on model-based fault diagnosis and fault tolerant with emphasis on networked control systems. The results of his research works are published in over 50 articles in journals and book contributions and 150 conference papers. Dr Sauter is currently serving as an associate editor for the journal of Applied Mathematics and Computer Science and senior editor for the Journal of Intelligent \& Robotic Systems. He has also been appointed by The IEEE control system society to the position of general chair for the organisation of the IEEE Multi Conference on system and control 2014 (MSC'14). 